\title{
Extension of Some Results for Channel Capacity Using a Generalized Information Measure
}

\author{
Aharon Ben-Tal ${ }^{1 *}$ and Marc Teboulle ${ }^{2}$ \\ ${ }^{1}$ Technion, Israel Institute of Technology, Haifa, Istael, and Department of Industrial and \\ Operations Engineering, University of Michigan, Ann Arbor, MI 48109, USA \\ ${ }^{2}$ Department of Mathematics, Statistics and Computing Science, Dalhousie University, \\ Halifax, Nova Scotia, Canada B3H $3 \mathbf{J} 5$ \\ Communicated by M. Zakai
}

\begin{abstract}
A new formulation for the channel capacity problem is derived by using the duality theory of convex programming. The simple nature of this dual representation is suitable for computational purposes. The results are derived in a unified way by formulating the channel capacity problem as a special case of a general class of concave programming problems involving a generalized information measure recently introduced by Burbea and Rao [10].
\end{abstract}

\section{Introduction}

Channel capacity, a basic concept in information theory, was introduced by Shannon [27] to specify the maximum rate at which information can be reliably conveyed by the channel. Roughly speaking, the basic theorem of information theory, the so-called "noisy channel coding theorem," states that if a given noisy channel has capacity $C$, it is possible to transmit, over this channel, messages of sufficiently large length and still be able to decode them with an arbitrary small probability for error, provided the rate of transmission is less than $C$. Methods for computing the capacity $C$ of a discrete channel have been studied by Muroga [21], Cheng [12], and Takano [28]. The best-known algorithm, however, is the one introduced independently by Arimoto [2] and Blahut [7]. A somewhat similar

\footnotetext{
* Research supported by National Science Foundation Grant No. ECS-8604354.
} 
iterative procedure based on the method of quasi-concave programming was proposed by Meister and Oettli [20]. In these previously mentioned works, the computational schemes are derived using the primal formulation of the channel capacity problem.

In this paper the classical channel capacity problem is embedded in a family $\left(P_{\varphi}\right)$ of linearly constrained concave programming problems, each member of which is determined by a choice of a convex function $\varphi$; the classical case corresponding to $\varphi(t)=t \log t$. The objective function in $\left(P_{\varphi}\right)$ is the generalized average mutual information measure, recently introduced by Burbea and Rao [10], and the optimal value of $\left(P_{\varphi}\right)$ is our generalized channel capacity $C_{\varphi}$.

A duality theory is developed for $\left(P_{\varphi}\right)$ resulting in a dual representation of $C_{\varphi}$. As a special case, a new formulation of the classical channel capacity is obtained. The dual of $\left(P_{\varphi}\right)$ (denoted $\left(D_{\varphi}\right)$ ) is a specially structured unconstrained minimax problem, thus rendering itself to efficient computational methods. The dual formulation is also very useful for obtaining upper bounds for $C_{\varphi}$. The paper is organized as follows. In Section 2 the formulation of the classical channel capacity problem is given and the iterative method of Arimoto [2] and Blahut [7] is briefly reviewed. In Section 3 we formulate the generalized capacity $C_{\varphi}$ and develop the theory leading to two different dual representations, the first (Theorem 3.1) is suitable for computations and the second (Corollary 3.1) is useful for deriving upper bounds. The bounds are given in Section 4, it is also shown there that the bound is attained for symmetric channels, i.e., an explicit formula for $C_{\varphi}$ is obtained. Section 5 contains concluding remarks and a brief discussion on possible extensions.

\section{The Channel Capacity Problem}

Consider a communication channel described by an input alphabet $A=$ $\{1,2, \ldots, m\}$, an output alphabet $B=\{1,2, \ldots, n\}$, and by a probability transition matrix $Q=\left\{Q_{k j}\right\}$, where $Q_{k j}$ is the probability of receiving the output letter $k \in B$ when input letter $j \in A$ was transmitted, i.e., $\sum_{k=1}^{n} Q_{k j}=1$ for all $j \in A$ and $Q_{k j} \geq 0$ for all $k \in B, j \in A$. The capacity of the channel is defined as

$$
C:=\max _{p \in \mathbb{P}_{m}} I(p, Q):=\max _{p \in \mathbb{P}_{m}} \sum_{j=1}^{m} \sum_{k=1}^{n} p_{j} Q_{k j} \log \frac{Q_{k j}}{\sum_{l=1}^{m} p_{l} Q_{K l}},
$$

where

$$
\mathbb{P}_{m}:=\left\{p \in \mathbb{R}^{m}: p_{j} \geqq 0, \forall j \in A, \sum_{j=1}^{m} p_{j}=1\right\}
$$

is the set of all discrete finite probability measures on the channel input, and $I(p, Q)$ is known as the average mutual information between the channel input and channel output, considered here as a function of $p$. The utility of the concept of capacity is widely discussed in the literature and for more details the reader is referred to Shannon [27], Gallager [15], and Jelineck [16], and to the more recent book of Csiszar and Korner [14]. For a given probability transition matrix 
$Q$, it is shown in Gallager [15] that $I(\cdot, Q)$ is a concave function of $p$ and therefore problem (2.1) is a concave programming problem over the simplex $\mathbb{P}_{m}$, then any of a number of readily available nonlinear programming codes can be used to compute $C$. However, as reported by Blahut [7], computational experience with nonlinear programming codes applied to problem (2.1) have proved to be inefficient even for small alphabet sizes and to be impractical for the larger alphabet sizes. This motivated Arimoto [2] and Blahut [7] to develop independently a systematic iterative method for computing the capacity. This was done by exploiting the special structure of the objective function $I(\cdot, Q)$. More specifically, let $P=\left(P_{j k}\right)$ denote a transition matrix from the channel output alphabet to the channel input alphabet, then

$$
I(P, Q)=\max _{P \in T}\left\{J(p, P ; Q):=\sum_{j=1}^{m} \sum_{k=1}^{n} p_{j} Q_{k j} \log \frac{P_{j k}}{p_{j}}\right\},
$$

where

$$
T:=\left\{P \in \mathbb{R}^{m} \times \mathbb{R}^{n}: \sum_{j=1}^{m} P_{j k}=1, \text { all } k=1, \ldots, n, P_{j k} \geq 0 \text { all } j \text { and } k\right\} .
$$

This can be verified by noting that the maximum of $J$ is attained at

$$
P_{j k}^{*}=\frac{Q_{k j} p_{j}}{\sum_{l=1}^{m} Q_{k l} p_{l}} .
$$

The Arimoto-Blahut algorithm can be summarized as follows:

(O) Choose an initial probability vector $p^{(0)} \in \mathbb{P}_{m}$.

At iteration $r$, where $p^{(r)}$ is given:

(i) Compute $P^{(r)}=\operatorname{argmax}_{P \in T} J\left(p^{(r)}, P ; Q\right)$.

(ii) Update $p^{(r+1)}=\operatorname{argmax}_{p \in P_{m}} J\left(p, P^{(r)} ; Q\right)$.

(iii) Iterate $r \leftarrow r+1$.

The solution of (2.4) is explicitly given by

$$
p_{j}^{(r+1)}=p_{j}^{(r)} \frac{c_{j}\left(p^{(r)}\right)}{\sum p_{j} c_{j}\left(p^{(r)}\right)},
$$

where for any $p \in \mathbb{P}_{m}$

$$
c_{j}(p)=\exp \left\{\sum_{k} Q_{k j} \log \frac{Q_{k j}}{\sum_{l} p_{l} Q_{k l}}\right\} .
$$

That the method (2.5) converges, i.e., $\lim _{r \rightarrow \infty} I\left(p^{(r)}, Q\right)=C$, see Arimoto [2] and Blahut [7]. The amount of computation involved depends upon the size of the channel matrix. Some conditions under which the amount of computation can be reduced are discussed in Cheng [12] and Takano [28].

In this paper we suggest a dual formulation to the channel capacity problem. The simple nature of this dual problem opens the possibility of applying many of the recent numerical schemes available in the mathematical programming literature, in particular for a large alphabet size (see e.g., [26]). This will be discussed in the next sections. 


\section{The Generalized Capacity Problem and Its Duals}

In this section we derive, in a unified way, a dual representation for the channel capacity problem. Recall that the channel capacity $C$ is given as the optimal value of the following optimization problem:

$$
\sup _{p \in \mathbb{P}_{m}} \sum_{j} \sum_{k} p_{j} Q_{k j} \log \frac{Q_{k j}}{\sum_{l=1}^{m} p_{l} Q_{k l}} .
$$

We denote by $q_{k}$ the output probabilities, then $q_{k}:=\sum_{j=1}^{m} p_{j} Q_{k j}, q_{k} \geq 0$ for all $k=1, \ldots, n, \sum_{k=1}^{n} q_{k}=1$, i.e., with our notation $q \in \mathbb{P}_{n}$. In the decision variables $\left(p_{j}, q_{k}\right)$, problem (3.1) can be written equivalently as

$$
\begin{gathered}
\text { (P) } \sup _{p \in \mathbb{P}_{m}} \sup _{q \in \mathbb{P}_{n}} \sum_{j} \sum_{k} p_{j} Q_{k j} \log Q_{k j}-\sum_{k=1}^{n} q_{k} \log q_{k} \\
\text { s.t. } \quad \sum_{j=1}^{m} p_{j} Q_{k j}=q_{k}, \quad \forall k=1, \ldots, n .
\end{gathered}
$$

The objective function in (3.2) is concave in $(p, q)$, therefore problem $(P)$ is a linearly constrained concave program. Also note that the feasible set of $(P)$ is a compact convex polyhedron in $\mathbb{R}^{m+n}$, hence the sup in $(P)$ is actually attained. The special structure of the objective function formulated in (3.2) (linear in $p$ minus strictly convex in $q$ ) motivated us to consider the following general class of concave programming problems:

$$
\begin{array}{r}
\left(P_{\varphi}\right) \max _{p \in \mathbb{P}_{m}} \max _{q \in \mathbb{P}_{n}} \sum_{j=1}^{m} p_{j} \sum_{k=1}^{m} \varphi\left(Q_{k j}\right)-\sum_{k=1}^{n} \varphi\left(q_{k}\right) \\
\text { s.t. } \quad \sum_{j=1}^{m} p_{j} Q_{k j}=q_{k}, \quad k=1, \ldots, n .
\end{array}
$$

Throughout the rest of this paper, we assume that $\varphi$ is a given twice continuously differentiable strictly convex function defined on an interval containing $(0,1]$, normalized such that $\varphi(0)=\varphi(1), \varphi^{\prime}(1)<\infty$, and satisfying the additional assumption $\lim _{t \rightarrow 0^{+}} \varphi^{\prime}(t)=-\infty$. Note that the latter assumption holds if $\varphi$ is essentially smooth in $[0,+\infty)$ (see, e.g., Rockafellar [24]). We denote the class of such $\varphi$ by $\Phi$, accordingly, $C_{\varphi}$ will denote the optimal value of problem $\left(P_{\varphi}\right)$. An important example of functions $\varphi \in \Phi$ is provided by the family $\Phi_{\alpha}$ of functions (parametrized by $\alpha$ ):

$$
\varphi_{\alpha}(t)= \begin{cases}\frac{1}{1-\alpha}\left(t-t^{\alpha}\right) & \text { for } 0<\alpha<1, \\ t \log t & \text { for } \alpha=1 .\end{cases}
$$

Clearly, with $\varphi_{1}(t)=t \log t$, problem $\left(P_{\varphi}\right)$ is just the classical channel capacity problem $(P)$.

The objective function used in $\left(P_{\varphi}\right)$ is exactly the generalized average mutual information measure introduced and studied by Burbea and Rao [10]. A related generalized measure of information was also recently introduced by Ben-Tal and 
Teboulle [6] and the associated rate distortion function was studied. For additional generalizations and applications of generalized information measures the reader is referred to Aczel [1], Arimoto [3], Burbea [8], [9], Burbea and Rao [11], Csiszar [13], Rao and Yayak [22], Renyi [23], and Ziv and Zakai [30].

The dual representation of $\left(P_{\varphi}\right)$ will be derived via Langrangian duality. Before stating the main result of this section we introduce the following notations and definitions. For any $\varphi \in \Phi$, let $f: \mathbb{R}^{n} \rightarrow \mathbb{R}$ be defined by

$$
f(v)=\inf _{n \in \mathbb{R}}\left\{\eta+\sum_{k=1}^{n} \varphi^{*}\left(v_{k}-\eta\right)\right\},
$$

where $\varphi^{*}(y)=\sup _{t}\{t y-\varphi(t)\}$ denotes the usual convex conjugate of $\varphi$. Also, let $l: \mathbb{R}^{n} \rightarrow \mathbb{R}$ be defined by

$$
l(v)=\max _{1 \leq j \leq m}\left\{l_{j}(v)+b_{j}\right\},
$$

where $\left\{l_{j}\right\}$ are the linear functions given by $l_{j}(v)=-\sum_{k=1}^{m} v_{k} Q_{k j}$ and $b_{j}$ denotes the constants $b_{j}=\sum_{k=1}^{m} \varphi\left(Q_{k j}\right)$.

Theorem 3.1. The dual problem of $\left(P_{\varphi}\right)$, for $\varphi \in \Phi$, is given by

$$
\left(D_{\varphi}\right) \inf _{v \in R^{n}}\{f(v)+l(v)\} .
$$

If $\left(P_{\varphi}\right)$ is feasible the minimum in $\left(D_{\varphi}\right)$ is attained and the optimal values coincide:

$$
C_{\varphi}=\max \left(P_{\varphi}\right)=\min \left(D_{\varphi}\right) .
$$

Proof. The Langrangian for problem $\left(P_{\varphi}\right)$ is

$$
L(p, q, v)=-\sum_{k=1}^{n} \varphi\left(q_{k}\right)+\sum_{j=1}^{m} p_{j} \sum_{k=1}^{n} \varphi\left(Q_{k j}\right)+\sum_{k=1}^{n} q_{k} v_{k}-\sum_{j, k} p_{j} v_{k} Q_{k j}
$$

and is separable in the decision variables $(p, q)$. The dual objective function is then

$$
h(v)=\max _{p \in \mathbb{P}_{m}} \sum_{j=1}^{m} p_{j}\left(\sum_{k=1}^{n} \varphi\left(Q_{k j}\right)-v_{k} Q_{k j}\right)+\max _{q \in \mathbb{P}_{n}} \sum_{k=1}^{n} q_{k} v_{k}-\varphi\left(q_{k}\right)
$$

and the dual problem is defined as $\min _{v \in \mathbb{R}^{n}} h(v)$. The first "max" in (3.8) is easily computed:

$$
\max _{p \in \mathbb{P}_{m}} \sum_{j} p_{j}\left(\sum_{k} \varphi\left(Q_{k j}\right)-v_{k} Q_{k j}\right)=\max _{1 \leq j \leq m}\left\{\sum_{k=1}^{n} \varphi\left(Q_{k j}\right)-v_{k} Q_{k j}\right\} .
$$

To evaluate the second "max" in (3.8) we note that a Langrangian dual of

$$
\alpha:=\max _{q \in \mathbb{P}_{n}} \sum_{k=1}^{n} q_{k} v_{k}-\varphi\left(q_{k}\right)
$$

is given by

$$
\beta:=\min _{\eta \in \mathbb{R}}\left\{\eta+\sum_{k} \max _{q_{k} \geq 0}\left\{q_{k}\left(v_{k}-\eta\right)-\varphi\left(q_{k}\right)\right\},\right.
$$


where $\eta$ is the Lagrange multiplier for the equation $\sum q_{k}=1$. Problem (3.10) is a linearly constrained concave program trivially satisfying the Slater constraint qualification and hence by standard duality arguments [23] we have $\alpha=\beta$.

To compute $\beta$, we consider the problem $e(y)=\sup _{t \geq 0}\{t y-\varphi(t)\}$. By the monotonicity of $\varphi^{\prime}$, it follows that

$$
e(y)= \begin{cases}\sup _{t \in \mathbb{R}}\{t y-\varphi(t)\}=\phi^{*}(y) & \text { if } y \geq \varphi^{\prime}(0), \\ -\xi(0) & \text { if } y<\varphi^{\prime}(0) .\end{cases}
$$

But since we assumed that $\varphi^{\prime}(0)=-\infty$, we conclude in fact that $e(y)=\varphi^{*}(y)$. Using this result in (3.11) we get

$$
\beta=\min _{\eta \in \mathbb{R}}\left\{\eta+\sum_{k=1}^{n} \varphi^{*}\left(v_{k}-\eta\right)\right\} .
$$

Substituting (3.9) and (3.12) in (3.8) and using the notations (3.5) and (3.6) we thus obtain $h(v)=f(v)+l(v)$ and hence the dual representation (3.7) is proved. Further, for $\varphi \in \Phi, \varphi$ is convex and then $\eta+\sum_{k} \varphi^{*}\left(v_{k}-\eta\right)$ is jointly convex in $(\eta, v) \in \mathbb{R} \times \mathbb{R}^{n}$, hence (by Theorem 1 of Rockafellar [25]) $f(v)$ is convex. Finally, since $\left(P_{\varphi}\right)$ is assumed feasible and is linearly constrained, the Slater regularity condition holds trivially and then it follows from standard duality results (see, e.g., [24]) that the $\inf \left(D_{\varphi}\right)$ is attained and $\max \left(P_{\varphi}\right)=\min \left(D_{\varphi}\right)$.

The next result shows how to obtain the optimal solution of the primal problem $\left(P_{\varphi}\right)$ from an optimal solution of the dual.

Theorem 3.2. Let $\bar{v}$ be the optimal solution of $\left(D_{\varphi}\right)$. Then the optimal solution $(\bar{p}, \bar{q})$ of the primal problem $\left(P_{\varphi}\right)$ is computed as follows:

$$
\bar{q}_{k}=\left(\varphi^{\prime}\right)^{-1}\left(\bar{v}_{k}-\bar{\eta}\right), \quad k=1, \ldots, n,
$$

where $\bar{\eta}$ is the unique solution of the equation

$$
\sum_{k}\left(\varphi^{\prime}\right)^{-1}\left(\bar{v}_{k}-\bar{\eta}\right)=1
$$

and $\bar{p}$ is the optimal solution of the linear program

$$
\begin{aligned}
\left(L_{\varphi}\right) \quad & \max \sum_{j} p_{j}\left(\sum_{k} \varphi\left(Q_{k j}\right)\right) \\
\text { s.t. } & \sum_{j} p_{j} Q_{k j}=\tilde{q}_{k}, \quad k=1, \ldots, n, \\
& \sum_{j} p_{j}=1, \quad p_{j} \geq 0, \quad j=1, \ldots, m .
\end{aligned}
$$

Proof. The expression (3.13) for $\bar{q}_{k}$ is just the optimality conditions for $q_{k}=\bar{q}_{k}$ to solve the inner maximization in (3.11) (recall that the optimal $q_{k}$ cannot be zero as explained in the proof of Theorem 3.1). The optimality condition for $\eta=\bar{\eta}$ to solve the convex unconstrained problem (3.12) is

$$
\sum_{k}\left(\varphi^{*}\right)^{\prime}\left(v_{k}-\eta\right)=1 \text {. }
$$


But $\left(\varphi^{*}\right)^{\prime}=\left(\varphi^{\prime}\right)^{-1}$ (see, e.g. Section 26 of [24]) and thus (3.15) is exactly (3.14). Now, since $\varphi \in \Phi, \varphi^{\prime}(0)=-\infty$ and $\varphi^{\prime}(1)<\infty$ implying $\left(\varphi^{\prime}\right)^{-1}(-\infty)=0$ and $\left(\varphi^{\prime}\right)^{-1}(\infty)>1$. Thus equation (3.14) has a solution $\bar{\eta}=\eta(\bar{v})$ for all $\bar{v}$, which is also unique since $\varphi^{\prime}$ (and hence $\left(\varphi^{\prime}\right)^{-1}$ ) is strictly monotone. The statement concerning $\bar{p}$ follows immediately from (3.4).

The dual problem (3.7) is an unconstrained discrete minimax problem. Many algorithms for such problems have been proposed in the nonsmooth optimization literature, see, e.g., Wolfe [29], Lemarechal [18], [19], and, more recently, Kiwiel [17]. Alternatively, the dual problem (3.7) can be reformulated as a linearly constrainted convex program in $\mathbb{R}^{n+1}$. Indeed, by defining $V_{n+1}:=$ $\max _{1 \leq j \leq n}\left\{l_{j}(v)+b_{j}\right\}$, problem (3.7) is equivalent to

$$
\min _{v \in \mathbb{A}^{n+1}} f(v)+v_{n+1}
$$

s.t. $l_{j}(v)+b_{j}-v_{n+1} \leq 0 \quad$ for all $j=1, \ldots, m$,

in which case many nonlinear programming codes are readily available for solving the dual formulation (3.16) (see, e.g., Schittowsky [26] or the recent generalized reduced gradient code of Lasdon).

The special structure of the dual problem

$$
\min _{v \in \mathbb{R}^{n}}\left\{f(v)+\max _{1 \leq j \leq m}\left\{l_{j}(v)+b_{j}\right\}\right\}
$$

suggests a method in which $f$ is approximated at the $r$ th iteration by a polyhedral function (i.e., pointwise maximum of finitely many affine functions) $\pi_{r}(v)$, and the next iteration point $v^{r+1}$ is the optimal solution of

$$
\min _{v}\left\{\pi_{r}(v)+\max \left\{l_{j}(v)+b_{j}\right\}\right\}
$$

Since (3.17) is a linear minimax problem it can be efficiently solved with simplexlike algorithms (e.g., [4] and [5]). The new polyhedral approximation $\pi_{r+1}(v)$ is obtained by

$$
\pi_{r+1}(v)=\max \left\{\pi_{r}(v), s_{r+1}(v)\right\},
$$

where $s_{r+1}(\cdot)$ is the affine support of $f$ at $v^{r+1}$.

Let us derive the dual representation of the classical channel capacity $C$. This is done simply by substituting $\varphi(t)=t \log t$ in problem $\left(D_{\varphi}\right)$. The conjugate is $\varphi^{*}\left(t^{*}\right)=e^{t^{*}-1}$ and so

$$
f(v)=\min _{\nu \in R}\left\{\eta+\sum_{k} e^{v_{k}-1}\right\}=\log \sum_{k=1}^{n} e_{k}^{v}
$$

by simple calculus.

Then, using Theorem 3.1, a dual representation of $C$ is given by

$$
C=\min _{v \in \mathbb{R}^{n}}\left\{\log \sum_{k=1}^{n} e^{v_{k}}+\max _{1 \leq j \leq m}\left\{\sum_{k} Q_{k j} \log Q_{k j}-\sum_{k} Q_{k j} v_{k}\right\}\right\} .
$$


In the last part of this section we present an alternative dual problem to $\left(P_{\varphi}\right)$ which is given directly in terms of the problem's data.

Corollary 3.1. Let $\varphi \in \Phi$, then

$$
C_{\varphi}=\min _{y \in \mathbb{P}_{n}} \max _{1 \leq j \leq m}\left\{\sum_{k} \varphi^{\prime}\left(y_{k}\right)\left(y_{k}-Q_{k j}\right)+\varphi\left(Q_{k j}\right)-\varphi\left(y_{k}\right)\right\} .
$$

Proof. From the proof of Theorem 3.2 it follows that $f(v)$ defined in (3.5) is given by

$$
f(v)=\eta(v)+\sum_{k} \varphi^{*}\left(v_{k}-\eta(v)\right),
$$

where $\eta(v)$ is the unique solution of the equation

$$
\sum_{k}\left(\varphi^{*}\right)^{\prime}\left(v_{k}-\eta\right)=1 \text {. }
$$

Define new variables $y_{k}=\left(\varphi^{*}\right)^{\prime}\left(v_{k}-\eta(v)\right)$. Then $\sum_{k} y_{k}=1$ and $y_{k} \geq 0$ since $\left(\varphi^{*}\right)^{\prime}(-\infty)=0$ and $\left(\varphi^{*}\right)^{\prime}$ is increasing, hence $y \in \mathbb{P}_{n}$. From the definition of $y_{k}$, and using $\left(\varphi^{*}\right)^{\prime}=\left(\varphi^{\prime}\right)^{-1}$,

$$
v_{k}-\eta(v)=\varphi^{\prime}\left(y_{k}\right)
$$

Substituting (3.21) in (3.7) and using (3.20) we obtain

$$
C_{\varphi}=\min _{v, y}\left\{\eta(v)+\sum \varphi^{*}\left(\varphi^{\prime}\left(y_{k}\right)\right)+\max _{1 \leq j \leq m} \sum_{k} \varphi\left(Q_{k j}\right)-Q_{k j}\left(\eta(v)+\varphi^{\prime}\left(y_{k}\right)\right)\right\} .
$$

But $\sum_{k} Q_{k j}=1$, hence

$$
C_{\varphi}=\min _{y}\left\{\sum_{k} \varphi^{*}\left(\varphi^{\prime}\left(y_{k}\right)\right)+\max _{1 \leq j \leq m} \sum_{k} \varphi\left(Q_{k j}\right)-Q_{k j} \varphi^{\prime}\left(y_{k}\right)\right\} .
$$

Finally, using in (3.22) a simple fact concerning conjugate functions,

$$
\varphi^{*}\left(\varphi^{\prime}(t)\right)=t \varphi^{\prime}(t)-\varphi(t)
$$

we get the desired result (3.19).

Applying Corollary 3.1 to the classical case $\varphi(t)=t \log t$, a little algebra shows that a dual representation of $C$ is

$$
C=\min _{y \in \mathbb{P}_{n}} \max _{1 \leq j \leq m} \sum_{k} Q_{k j} \log \frac{Q_{k j}}{y_{k}}
$$

and we recover here a result given in Meister and Oettli [20].

\section{Upper Bound for $C_{\varphi}$}

The dual representation derived in Corollary 3.2 is also useful for deriving an upper bound on $C_{\varphi}$. 
Theorem 4.1. For any $\varphi \in \Phi$,

$$
C_{\varphi} \leq \sum_{k}\left\{\varphi^{\prime}\left(\sum_{j} \frac{Q_{k j}}{m}\right) \sum_{j} \frac{Q_{k j}}{m}-\varphi\left(\sum_{j} \frac{Q_{k j}}{m}\right)\right\}+\max _{1 \leq j \leq m} \sum_{k} \varphi\left(Q_{k j}\right)-Q_{k j} \varphi^{\prime}\left(\sum_{j} \frac{Q_{k j}}{m}\right) .
$$

Proof. From the dual representation (3.19)

$$
C_{\varphi} \leq \max _{1 \leq j \leq m}\left\{\sum_{k=1}^{n} \varphi^{\prime}\left(y_{k}\right)\left(y_{k}-Q_{k j}\right)+\varphi\left(Q_{k j}\right)-\varphi\left(y_{k}\right)\right\}
$$

for every $y$ satisfying $\sum_{k=1}^{n} y_{k}=1$ and $y_{k} \geq 0, k=1, \ldots, n$, in particular, for $y_{k}=$ $\left(\sum_{j=1}^{m} Q_{k j}\right) / m$. Substituting this special choice in (4.2), and rearranging the terms, we obtain the upper bound in the theorem.

Example 4.1. Let us consider the family $\Phi_{\alpha}$ with functions $\varphi_{\alpha}$ given by

$$
\varphi_{\alpha}(t)= \begin{cases}\frac{1}{1-\alpha}\left(t-t^{\alpha}\right), & 0<\alpha<1, \\ t \log t, & \alpha=1 .\end{cases}
$$

We denote respectively by $C_{\alpha}$ and $U_{\alpha}$ the corresponding capacity and its upper bound. Using Theorem 4.1 we get

$$
C_{\alpha} \leq U_{\alpha}
$$

with

$$
U_{\alpha}= \begin{cases}\frac{1}{m^{\alpha}} \sum_{k}\left(\sum_{j} Q_{k j}\right)^{\alpha}+\frac{1}{1-\alpha} \max _{1 \leq j \leq m} \frac{\alpha}{m^{\alpha}-1} \sum_{k} Q_{k j}\left(\sum_{j} Q_{k j}\right)^{\alpha-1}-\sum_{k} Q_{k j}^{\alpha} \\ \log m+\max _{1 \leq j \leq m} \sum_{k} Q_{k j} \log \frac{Q_{k j}}{\sum_{l} Q_{k l}} & \text { if } 0<\alpha<1,\end{cases}
$$

In particular, we see that the classical lower bound derived in Arimoto [2] is recovered, i.e.,

$$
C \equiv C_{1} \leq U_{1}
$$

An interesting special case for which the upper bound is attained is in the case of a symmetric channel, i.e., one with the same set of entries in columns and rows of $Q$, with possible permutations. In that case we have $\sum_{j=1}^{m} Q_{k j}=$ const. $=\delta$ for all $k$.

Theorem 4.2. If the channel is symmetric, then, for any $\varphi \in \Phi, C_{\varphi}$ is equal to the upper bound, i.e.,

$$
C_{\varphi}=-n \varphi\left(\frac{1}{n}\right)+\frac{1}{m} \sum_{j, k} \varphi\left(Q_{k j}\right)
$$


Proof. From the primal formulation of $C_{\varphi}$ (see (3.4)) a lower bound is given by

$$
C_{\varphi} \geq-\sum_{k} \varphi\left(q_{k}\right)+\sum_{j=1}^{m} p_{j} \sum_{k=1}^{n} \varphi\left(Q_{k j}\right)
$$

for any $(p, q) \in \mathbb{P}_{m} \times \mathbb{P}_{n}$ satisfying

$$
\sum_{j} p_{j} Q_{k j}=q_{k}, \quad k=1, \ldots, n .
$$

Since the channel is symmetric, $\sum_{j} Q_{k j}=\delta$ for all $k$ and this implies that $m=n \delta$. Thus, $p_{j}^{*}=1 / m$ and $q_{k}^{*}=1 / n$ satisfy (4.5). Substituting $\left(p^{*}, q^{*}\right)$ in (4.4) we obtain the lower bound

$$
C_{\varphi} \geq-n \varphi\left(\frac{1}{n}\right)+\frac{1}{m} \sum_{j, k} \varphi\left(Q_{k j}\right) .
$$

From Theorem 4.1, using the fact $m=n \delta$, we have the upper bound

$$
C_{\varphi} \leq-n \varphi\left(\frac{1}{n}\right)+\max _{j} \sum_{k} \varphi\left(Q_{k j}\right) .
$$

Since the channel is symmetric, it has the same set of entries in each column, thus $\sum_{k} \varphi\left(Q_{k j}\right)=\gamma_{j}=$ const. for all $j$ and, hence,

$$
\max _{1 \leq j \leq m} \sum_{k} \varphi\left(Q_{k j}\right)=\frac{1}{m} \sum_{j, k} \varphi\left(Q_{k j}\right) .
$$

Therefore, substituting (4.8) in (4.7), we see that the upper bound for $C_{\varphi}$ coincides with its lower bound given in (4.6).

Example 4.2. Consider the Binary Symmetric Channel (BSC) defined by

$$
Q=\left[\begin{array}{cc}
1-\beta & \beta \\
\beta & 1-\beta
\end{array}\right] \text {. }
$$

Using Theorem 4.2 we obtain, for any $\varphi \in \Phi$,

$$
C_{\varphi}^{\mathrm{BSC}}=-2 \varphi\left(\frac{1}{2}\right)+\varphi(1-\beta)+\varphi(\beta) .
$$

In particular, for $\varphi(t)=t \log t$ we get the well-known result (see, e.g., [15] and [16])

$$
C^{\mathrm{BSC}}=\log 2+(1-\beta) \log (1-\beta)+\beta \log \beta .
$$

\section{Conclusions and Extension}

A new formulation of the channel capacity problem has been obtained by using the duality theory of convex programming. This new dual representation seems useful for computational purposes and the derivation of bounds. Furthermore, the results in this paper demonstrate that the new information measure of Burbea and Rao [10] can be successfully used to develop a generalized channel capacity theory. 
Finally, we remark that our duality framework can be easily extended to the multiple constrained channel capacity problem (i.e., which include additional linear inequality constraints on the input probability $p$, see [5]) to produce a simple dual formulation. Also, at the price of some additional technicalities, the continuous alphabet channel problem [7] [15] can be considered, via a duality theory for infinite-dimensional optimization problems framework, to obtain the continuous version of Theorem 3.1 and its corollary.

\section{References}

1. Aczel $\mathbf{J}$ (1984) Measuring information beyond communication theory-why some generalized information measures may be useful, others not. Aequationes Math 27:1-19.

2. Arimoto $S$ (1972) An algorithm for computing the capacity of arbitrary discrete memoryless channels. IEEE Trans. Inform Theory 18:14-20

3. Arimoto $S$ (1977) Information measures and capacity of order $\alpha$ for discrete memoryless channels. In: Csiszar I, Elias P (eds) Topics in Information Theory. North-Holland, Amsterdam, pp 41-52

4. Armstrong RD, Godfrey JR (1979) Two linear programming algorithms for the discrete $l_{1}$ norm problem. Math Comp 33:289-300

5. Barrodale I, Roberts FDK (1973) An improved algorithm for discrete $l_{1}$-linear approximation. SIAM J Numer Anal 10:839-848

6. Ben-Tal A, Teboulle M (1986) Rate distortion theory with generalized information measures via convex programming duality. IEEE Trans Inform Theory 32:630-641

7. Blahut RE (1972) Computation of channel capacity and rate distortion functions. IEEE Trans Inform Theory 28:489-495

8. Burbea J (1983) $J$-Divergence and related topics. Encyclopedia of Statistical Science vol. 4. Wiley, New York, pp 290-296

9. Burbea $\mathrm{J}$ (1984) The Bose-Einstein entropy of degree $\alpha$ and its Jensen difference. Utilitas Math $25: 225-240$

10. Burbea J, Rao CR (1982) On the convexity of some divergence measures based on entropy functions. IEEE Trans Inform Theory 28:489-495

11. Burbea J, Rao CR (1982) Entropy differential metric, distance and divergence measures in probability spaces-a unified approach. J Multivariate Anal 12:575-596

12. Cheng MC (1979) On the computation of capacity of a discrete memoryless channel. Inform and Control 24:292-298

13. Csiszar I (1967) Information-type measures of difference of probability distributions and indirect observations. Studia Sci Math Hungar 2:299-318

14. Csiszar I, Korner J (1981) Information Theory: Coding Theorems for Discrete Memoryless systems. Academic Press, New York

15. Gallager RG (1968) Information Theory and Reliable Communication. Wiley, New York

16. Jelinek F (1968) Probabilistic Information Theory. McGraw-Hill, New York

17. Kiwiel KC (1985) Methods of Descent for Nondifferentiable Optimization. Lecture Notes in Mathematics, Vol 1133. Springer-Verlag, Berlin.

18. Lemarechal C (1975) An extension of Davidon methods to nondifferentiable problems. Math. Programming Stud 3:95-109

19. Lemarechal C (1980) Nondifferentiable optimization. In: Dixon, Spedicato, Szego (eds) Nonlinear Optimization Theory and Algorithms. Birkhauser, Boston

20. Meister B, Oettli W (1967) On the capacity of a discrete constant channel. Inform and Control $11: 341-351$

21. Muroga S (1953) On the capacity of a discrete channel. J. Phys Soc Japan 8:484-494

22. Rao CR, Nayak TK (1985) Cross entropy, dissimilarity measures, and characterizations of quadratic entropy. IEEE Trans Inform Theory 31:589-593 
23. Renyi A (1961) On measures of entropy and information. Berkeley Symposium on Mathematical Statistics and Probability, vol. 1. University of California Press, Berkeley, pp 547-561

24. Rockafellar RT (1970) Convex Analysis. Princeton University Press, Princeton, NJ

25. Rockafellar RT (1974) Conjugate Duality and Optimization. Regional Conference Series in Applied Mathematics, no 16. SIAM, Philadelphia

26. Schittowsky K (1980) Nonlinear Programming Codes. Lecture Notes in Economics and Mathematical Systems, vol. 183. Springer-Verlag, Berlin

27. Shannon CE (1948) A mathematical theory of communication. Bell Syst Tech J 27:379-423 and $623-565$

28. Takano S (1975) On a method of calculating the capacity of a discrete memoryless channel. Inform and Control 29:327-336

29. Wolfe P (1975) A method of conjugate subgradients for minimizing nondifferentiable convex functions functions. Math. Programming Stud 3:145-173

30. Ziv J, Zakai M (1973) On functionals satisfying a data-processing theorem. IEEE Trans Inform Theory 19:275-283

Accepted 2 February 1987 